\title{
Clinical shorts
}

Oral laquinimod for multiple sclerosis: Oral laquinimod slows the progression of disability and reduces the rate of relapse in patients with relapsing-remitting multiple sclerosis. This is the conclusion of a multicentre, placebo-controlled trial in which over 1100 adults with relapsingremitting multiple sclerosis were randomly assigned to receive oral laquini$\bmod (0.06 \mathrm{mg}$ daily) or placebo for 24 months. There was a modest reduction in the mean annualized relapse rate in those receiving active treatment compared with placebo $(0.30$ v. 0.39 , risk ratio $0.77,95 \%$ confidence interval [CI] 0.65 to 0.91 ) and a reduction in the risk of disability progression $(11.1 \%$ v. $15.7 \%$, hazard ratio $0.64,95 \%$ CI 0.45 to 0.91 ). The laquinimod group also experienced fewer new or enlarged lesions seen on brain magnetic resonance imaging. Transient elevation of liver enzymes was more common in the treatment group. See $N$ Engl J Med 2012;366:1000-9.

Failure rates of metal-on-metal hip replacements: Metal-on-metal hip replacements failed at high rates in a study of over 400000 primary hip replacements in England and Wales between 2003 and 2011. Data from the National Joint Registry of England and
Wales showed that these devices performed less well than other types (see Figure). Failure was related to larger head size of the metal-on-metal devices (five-year revision rates $3.2 \%$, 95\% confidence interval $[\mathrm{CI}] 2.5$ to 4.1 for $28 \mathrm{~mm}$ head and $5.1 \%, 95 \%$ CI 4.2 to 6.2 for $52 \mathrm{~mm}$ head in 60-year-old men). Younger women were more likely to have their metal-on-metal implants revised. The authors suggest that patients with metal-on-metal implants be assessed regularly for implant failure and potential complications related to metal exposure. See Lancet 2012;DOI: 10.1016/S0140-6736(12)60353-5.

\section{Preventing stroke in patent foramen} ovale: Closure of patent foramen ovale with a device does not provide greater benefit than medical therapy alone in preventing recurrent stroke. A multicentre, randomized open-label trial included 909 adults who were randomized to receive closure with a percutaneous device plus antiplatelet therapy or medical therapy alone (warfarin or aspirin or both). All participants had a patent foramen ovale and had experienced an ischemic stroke or transient ischemic attack (TIA) within the previous six months. Implantation of the closure device was successful in

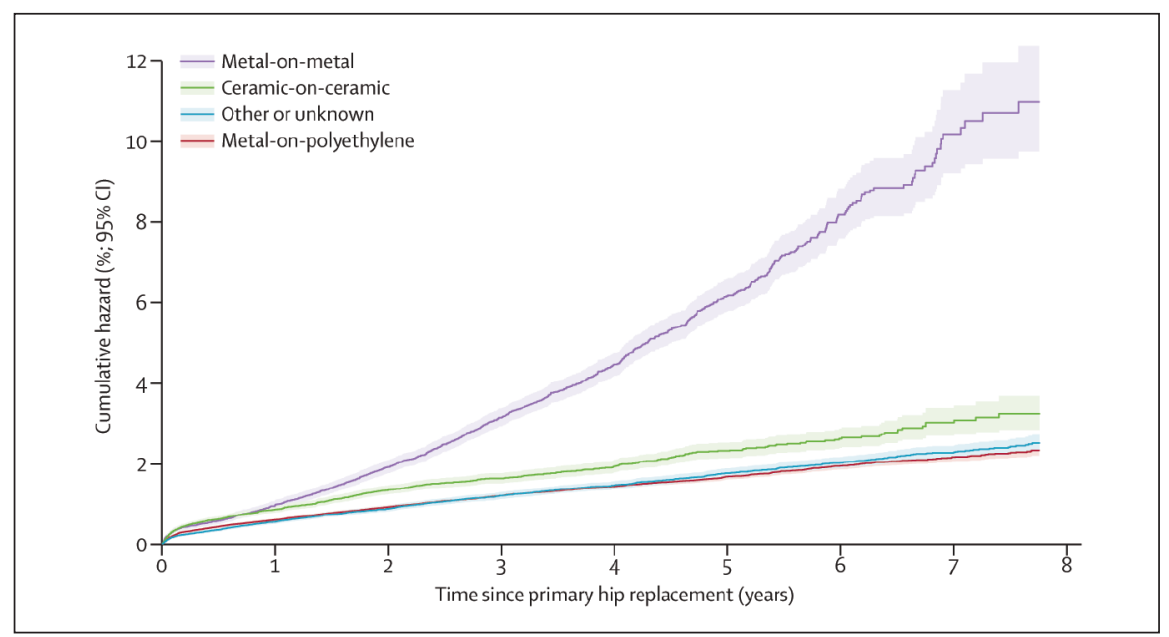

Reprinted from The Lancet, Smith et al. Failure rates of stemmed metal-on-metal hip replacements: analysis of data from the National Joint Registry of England and Wales, March 13, 2012, DOI:10.1016/S0140-6736(12)60353-5, with permission from Elsevier. $\mathrm{Cl}=$ confidence interval.
$89.4 \%(362 / 405)$ patients in the closure group, with effective closure (i.e., no or minor residual shunt) of $86.7 \%$ at two years. The cumulative incidence of stroke, TIA or death from neurologic causes during two years of follow-up, or death from any cause at 30 days was $5.5 \%$ in the closure group and $6.8 \%$ in the medical therapy group (hazard ratio $0.78,95 \%$ confidence interval 0.45 to 1.35). Atrial fibrillation was more common in the closure group. See $N$ Engl J Med 2012;366:991-9.

Discontinuation of antidepressants in dementia with neuropsychiatric symptoms: Discontinuation of antidepressants in patients with dementia and neuropsychiatric symptoms results in an increase in depressive symptoms. Because evidence for use of antidepressants to treat neuropsychiatric symptoms in dementia is limited, a double-blind, randomized, placebo-controlled trial studied the effects of discontinuing selective serotonin reuptake inhibitors (SSRI) in patients who had dementia and neuropsychiatric symptoms (e.g., delusions, agitation, apathy) and who lived in nursing homes. Those with a history of depression and schizophrenia were excluded. Participants $(n=128)$ were randomized to discontinuation of their medication over one week (replaced by placebo) or continuation of usual medication for a total of 25 weeks. At the end of the study, the discontinuation group had significantly higher scores on a validated depression scale than those who stayed on their medication (difference $-2.89,95 \%$ confidence interval -4.76 to $-1.02)$. The drop-out rate in the study was high (37\%), but withdrawals related to increased neuropsychiatric symptoms were more common in the discontinuation group. See BMJ 2012;344:e1566 doi: 10.1136/bmj.e1566.

\section{Diane Kelsall MD MEd \\ Deputy Editor, Clinical Practice CMAJ}

CMAJ 2012. DOI:10.1503/cmaj.120533 